\title{
Recurrent Transient Osteoporosis during Pregnancy and Treatment with Oral Bisphosphonates: A Case Report
}

\author{
Pavlos Sachsanidis, Mario Valerio Tartagni*, Urs Graf \\ Clinic of Gynecology and Obstetrics, Spital Linth, Uznach, Switzerland \\ Email: *mv.tartagni@libero.it
}

How to cite this paper: Sachsanidis, P., Tartagni, M.V. and Graf, U. (2017) Recurrent Transient Osteoporosis during Pregnancy and Treatment with Oral Bisphosphonates: A Case Report. Open Journal of Obstetrics and Gynecology, 7, 1044-1051. https://doi.org/10.4236/ojog.2017.710105

Received: August 28, 2017

Accepted: October 8, 2017

Published: October 11, 2017

Copyright (c) 2017 by authors and Scientific Research Publishing Inc. This work is licensed under the Creative Commons Attribution International License (CC BY 4.0).

http://creativecommons.org/licenses/by/4.0/

\begin{abstract}
Transient osteoporosis of the hip ( $\mathrm{TOH})$, also known as "Bone marrow edema Syndrome", is a rare disorder mainly affecting pregnant women in their third trimester, as well as middle-aged, overweight men. A 30-year-old Caucasian female G2P2, with history of transient osteoporosis of both ankles and C-Section during the last pregnancy in 2011, presented progressively severe bilateral hip pain with onset already in the $12^{\text {th }}$ gestational week. Imaging of the pelvis and bilateral hips with MRI obtained 6 days after the C-Section demonstrated bilateral bone-marrow edema of the hips. The patient was treated with a monthly single dose of $150 \mathrm{mg}$ ibandronate acid per os, for 3 months and physiotherapy. Repeated MRI performed 5 months postpartum revealed a complete remission of the disease. In contrast to the first onset of transient osteoporosis during the first pregnancy, which was only treated conservatively without bisphosphonates, the remission of the disease and patient's recovery with oral ibandronate therapy showed to be 4 months shorter. This case is unique in literature for both describing the onset of this rare disease twice in the same patient as well as its oral therapeutic approach.
\end{abstract}

\section{Keywords}

Transient Osteoporosis, Pregnancy, Oral Treatment, Bisphosphonates

\section{Introduction}

Bone marrow edema syndrome occurs with an incidence of 4:1,000,000 pregnant women per year, manifesting itself mainly in the last trimester or in the early postpartum phase [1]. This disorder is a self-limiting skeletal disease that is both reversible and painful. The most common affected site is the hip joint, while 
bilateral involvement occurs in circa $25 \%$ - 30\% of cases [2]. Suggested etiologies include pelvic nerve compression, vascular insufficiency or change in fibrinolytic system with pregnancy, still a definite cause remains unknown up to this date [3].

Generally, the first symptom is the sudden onset of pain, without a prior history of trauma that could explain it. Mild limitation of motion-range of the hip is frequently noted, with increasing pain through turning movements or weight bearing and easing with rest. The pain increases gradually over weeks or month and can eventually become disabling. The patient usually refers pain at the lateral aspect of the hip, in front of the thigh, the buttocks or the groin [4] [5].

Regarding sensitivity and specificity, MRI is the gold standard for the diagnosis, which in contrast to plain radiograph can also distinguish between a $\mathrm{TOH}$ and osteonecrosis. Other techniques such as bone scintigraphy could integrate the MRI findings, although not essential for the diagnosis [6].

\section{Case Report}

The patient, a 30-year-old Caucasian G2P2, with no history of trauma or systemic disease except a previous onset of transient osteoporosis of both ankles in the first pregnancy $\left(32^{\text {nd }}-33^{\text {rd }}\right.$ week), experienced during the first trimester $\left(12^{\text {th }}\right.$ week) of the second pregnancy a new and early onset of transient osteoporosis, this time involving both hips.

During this pregnancy, in year 2016, the pain presented itself suddenly and bilaterally localized at the hip and aggravated by weight bearing activities. The patient was only comfortable at rest. Movements concerning the right hip such as flexion, external and internal rotation were, due to the pain, barely possible. At the left hip the movements limitation concerned flexion and internal rotation, with time the pain progressively worsened, reaching by the $30^{\text {th }}$ gestational week a threshold such (9 points on the Visual Analogue Scale-VAS) that the patient could find no relief by analgesic therapy and physiotherapy and deambulation was only possible with the help of a crutches. The patient delivered electively at 38 2/7 gestational week by caesarean section to avoid possible hip fractures during a vaginal delivery and a uterine rupture due to the previous C-Section in 2011. The child was a boy and weighed $3610 \mathrm{~g}$. Three days after the operation an intra-articular puncture was performed and the analysis of the synovial fluid was negative for inflammation markers. Also, systemic inflammatory parameters and leucocytes in blood were in normal range. Given these findings at the $6^{\text {th }}$ postoperative day, a MRI was performed, which revealed evidence of a bilateral bone marrow edema in the head and neck of the femoral bone with a small joint effusion (Figure 1).

The patient was discharged a week after the cesarean section and promptly treated with a monthly single dose of $150 \mathrm{mg}$ ibandronate acid per os, for 3 months and physiotherapy. In addition, Vitamin D and Calcium supplementation were also administered. Breastfeeding was interrupted before ibandronate 


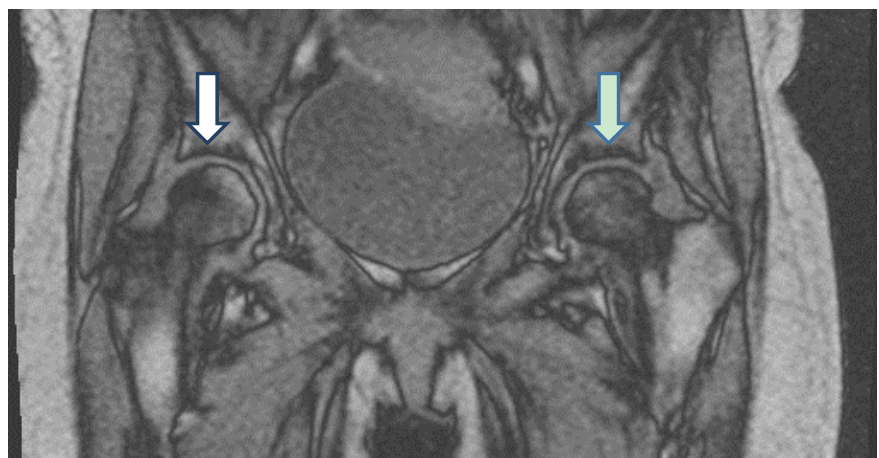

(a)

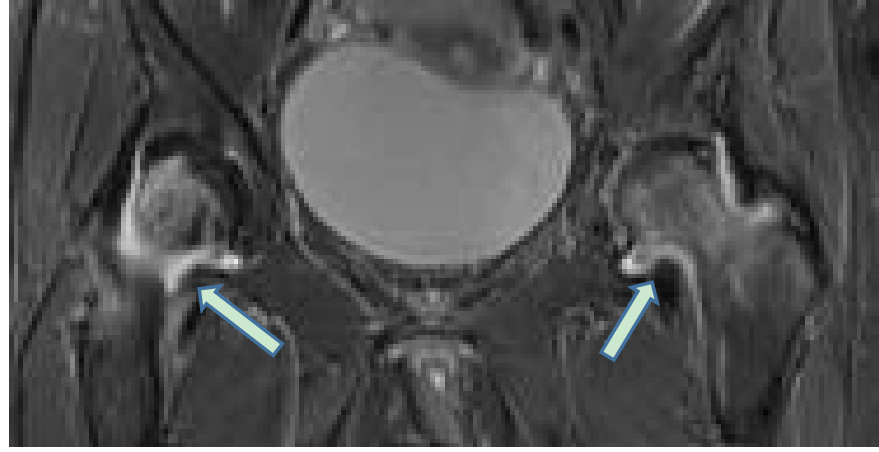

(b)

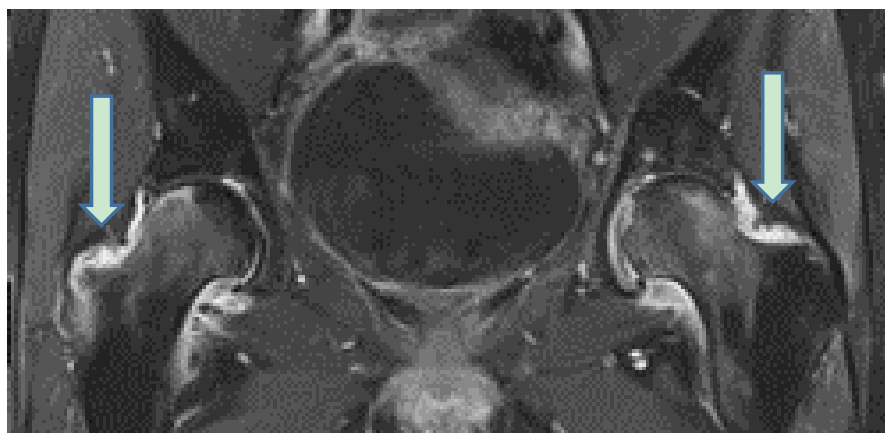

(c)

Figure 1. (a)-(c) Magnetic resonance images (MRI) of the pelvis showing a diffuse and bilateral epi- and metaphyseal bone marrow edema of the head and neck of femur and less pronounced in the acetabulum on the left side, a sclerotic area of $11 \mathrm{~mm}$ in the posterior column of the left acetabulum as well as a small-volume bilateral joint effusion. Response: Pregnancy-associated bilateral transient bone marrow edema of the hip joints and small-volume joint effusion on both sides.

acid administration and no more continued due to the risk of further bone demineralization. At the end of the $1^{\text {st }}$ postpartum month, the patient refers that the pain has still the same intensity ( 9 points VAS). Meanwhile she did not report any medication related-side effects, except tiredness. At the end of the $3^{\text {rd }}$ postpartum month the patient could walk without crutches due to an improvement of the pain (7 points VAS). A month later she noticed a further improvement of the condition ( 5 points VAS) and a new MRI was taken ( $5^{\text {th }}$ month postpartum) which showed a complete remission of the disease (Figure 2). 


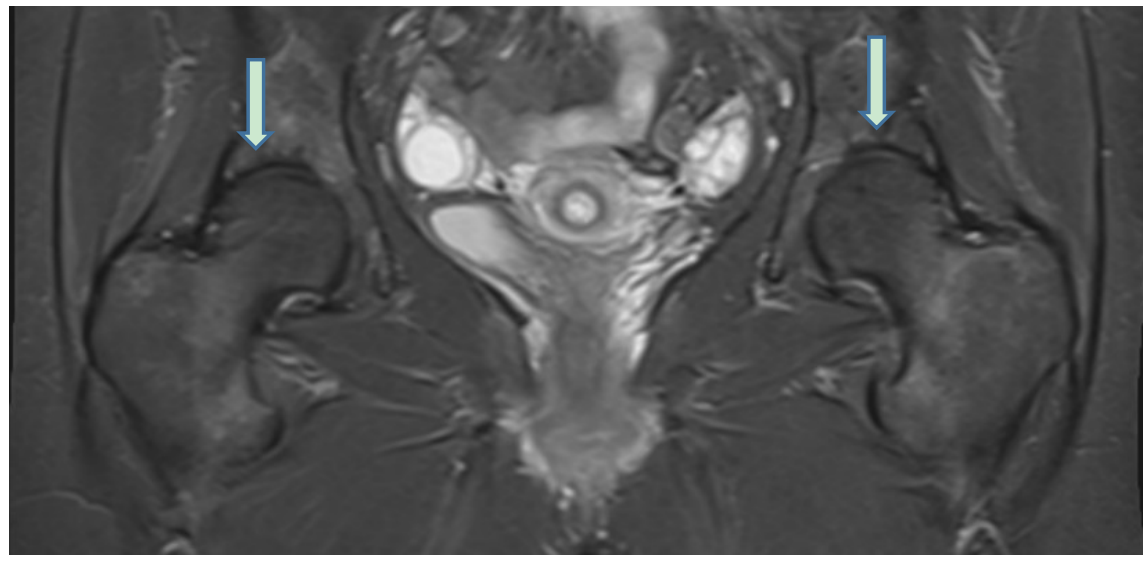

(a)

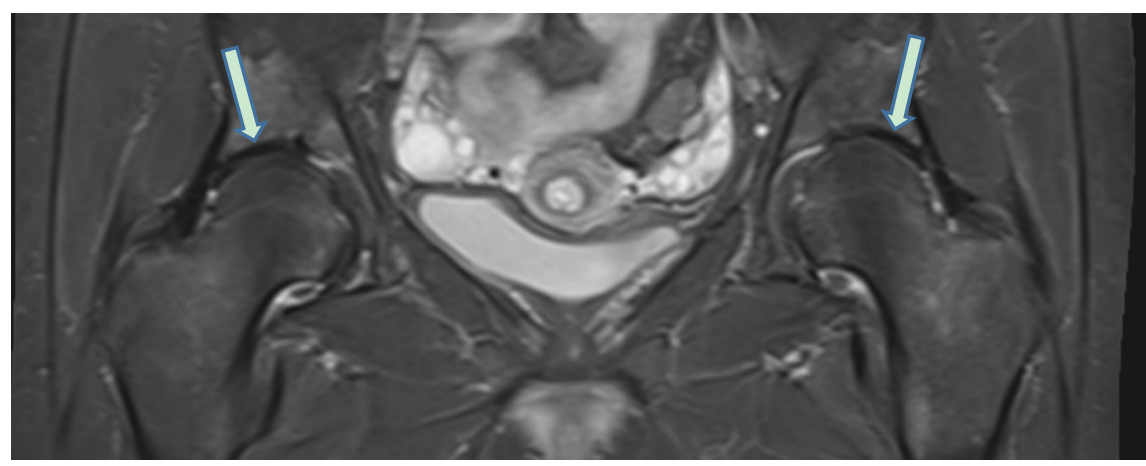

(b)

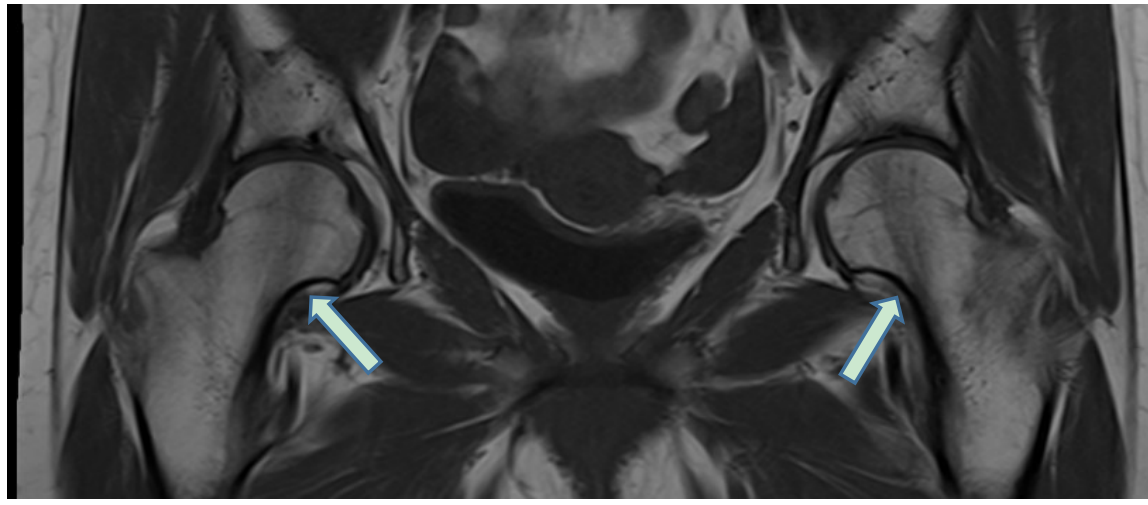

(c)

Figure 2. (a)-(c) A repeated MRI 5 months postpartum shows a complete remission of the bone marrow edema in both proximal femurs with now correct bone marrow signal.

The patient refers occasionally only little or no pain ( 0 - 3 points VAS) on both hips 6 months after the beginning of the therapy with ibandronate acid.

In the year 2011 a secondary caesarean section at 41 1/7 gestational weeks was performed due to pathological CTG after labor induction with misoprostol. At that time an MRI performed during the 32th gestational week showed a bone marrow edema involved prevalently both the lower legs and right foot (cuboidal and navicular bones) preventing the patient from walking without assistance.

The disease was managed conservatively with physiotherapy, Vitamin D, 
Calcium, compression stockings and subcutaneous enoxaparin $1 \times 1$ for 3 weeks after the operation due to difficult mobilization of the patient ( 9 points VAS). The patient wished at that time not to undergo a bisphosphonates therapy and not to interrupt breastfeeding. Physiotherapy and analgesic therapy (when necessary) lasted till the patient was completely healed. After two months the clinical picture was slightly better, the patient had still pain (8 - 7 points VAS) but she could walk slowly and the edema in the lower legs and feet disappeared. During the $5^{\text {th }}$ month postpartum, due to a prolonged healing course of the right foot and ankle, a control-MRI was taken showing a light improvement of the bone marrow edema. 9 months after delivery the patient had still light pain on the right ankle ( 3 points VAS) and could go back to work only a month after (0 points VAS).

\section{Discussion}

This condition was described for the first time by Curtiss and Kincaid in 1959 as "transient demineralization" [7], however Lequesne in 1968 used the term "transient osteoporosis" for the first time [8]. Other terms that have been used include "bone marrow edema" due to the MRI findings early in the progression of the disorder [9].

Suggested etiologies include pelvic nerve compression, vascular insufficiency or change in fibrinolytic system with pregnancy, but a definitive cause remains unknown [3]. Pathogenetically it is also discussed an alteration of the bone-metabolism during pregnancy and breastfeeding period. As known, a transfer of 30 g. of calcium takes place from the mother to her child during the pregnancy, of which $80 \%$ occurs in the third trimester, moreover circa $40 \mathrm{~g}$. will be transferred during breastfeeding. The source of calcium is the skeletal bones of the mother together with increased intestinal calcium absorption. In addition to this, other factors can play a role to increase the risks for a $\mathrm{TOH}$ : primary and secondary hyperparathyreoidismus, hypothyreose, disorders of Vit. D metabolism, chronic intestinal inflammatory diseases, osteomalacia, glucocorticoids, heparin, phenythoin, immobilization and genetic predisposition [1].

TOH occurs in three phases: 1) Stage 1 (first $1-2$ months) features a rapid increase in pain and functional disability but negative radiographic findings; 2) Stage 2 (after 2 - 3 months) features maximal symptoms and signs, with osteopenia noted on radiographs; 3) Stage 3 (up to 6 months) involves gradual regression of the disease and radiographic changes, the latter taking longer [2] [6].

Differential diagnoses include: avascular bone necrosis, reflex sympathetic dystrophy, stress fracture of the femoral neck, inflammatory arthritis, septic arthritis, synovial disorders (pigmented villonodular synovitis, synovial chondromatosis) and neoplasia [6] [7] [8] [9] [10].

MRI is the gold standard for the diagnosis, showing already the bone marrow edema in the first 48 hours after the onset of symptoms and documenting its resolution in the following 6 - 8 months [2]. Moreover, MRI allows also to distinguish 
between TOH and osteonecrosis, which can present similarly to TOH [6]. Our patient's findings on MRI were consistent with distinct MRI features of TOH in pregnancy. These findings were also supported by a negative intra-articular puncture done 3 days before it.

Before the advent of MRI, radionuclide bone scans were used for the diagnose of $\mathrm{TOH}$ due to their higher sensitivity in comparison to X-rays. Radionuclide bone scans are also able to differentiate between $\mathrm{TOH}$ and avascular necrosis as the uptake is more pronounced in $\mathrm{TOH}$ and there is no evidence of "cold spots" which are usually seen in avascular necrosis [11].

Given the benign course of the disease, prevention of damage to bones weakened by osteoporosis is crucial; the most severe possible complication is stress fracture of the hip [4]. In our case a C-section was performed in the $38^{\text {th }} 2 / 7$ gestational week in order to avoid a possible stress fracture of the hip during a vaginal delivery and a risk of uterine rupture due to the previous C-section 5 years before. In 2011, the pain involved predominantly both ankles and the right foot, thus a vaginal delivery was at that time not contraindicated. After induction of labor with misoprostol a C-section was anyway performed due to pathological CTG.

Treatment is mainly conservative, including reduction of weight bearing activities, analgesia, and physiotherapy [2]. Calcitonin, prednisolone and the bonesparing steroid, as well as deflazacort have also been used. Ibandronate, clodronate, pamidronate, neridronate and alendronate have all been used successfully as intramuscular or intravenous injections in conjunction with calcium and vitamin D supplements in patients with transient osteoporosis [12]. The use of bisphosphonates during pregnancy remains controversial. Although bisphosphonates are known to cross the placental barrier and may have an effect on fetal serum calcium levels and growth there have been no reports of congenital abnormalities associated with use of bisphosphonates in animal teratology studies [13]. Concerning bisphosphonates therapy during breastfeeding, a study showed that pamidronate is undetectable in breast milk of nursing mothers in the first $48 \mathrm{~h}$ after a $30 \mathrm{mg}$ infusion [14]. In our case, we decided to postpone the bisphosphonate therapy after the C-section and, in accordance with the patient, to suspend breastfeeding in order to avoid further bone demineralization. This strategy, in contrast to the non-pharmacological and non-breastfeeding-interruption approach during the previous pregnancy, led to a significant shortening of the course of the disease and patient's recovery.

The case report shown above is unique in the literature, in comparison to the other reported cases [2] [5] [7], for two reasons. First of all, the patient is suffering from this rare disease twice, by the first and second gestation. Moreover, the approach of the treatment with oral bisphosphonates, instead of intramuscular or intravenous, is not described earlier in the literature.

\section{Conclusion}

Ibandronate acid therapy per os is showed to be effective in shortening the 
course of transient osteoporosis postpartum and the patient's recovery of several months when compared with the non-bisphosphonate approach applied after the first pregnancy in the same patient. Therefore, we suggest it in addition to conservative and supportive treatment with physiotherapy, analgesic therapy, Vit. D and Calcium supplementation. Furthermore, we suggest also breastfeeding interruption in order to prevent further bone demineralization.

\section{Disclosure}

The authors declare that there is no conflict of interest regarding the publication of this paper.

\section{References}

[1] Cabrera, L. and Schreier, J. (2011) Schwangerschaftsassoziierte Osteoporose (SAOP) und transiente Osteoporose (TOP) der Hüfte in der Schwangerschaft. Geburtshilfe Frauenheilkd, 71, B12. https://doi.org/10.1055/s-0031-1295380

[2] Ma, F.Y. and Falkenberg, M. (2006) Transient Osteoporosis of the Hip: An Atypical Case. Clinical Orthopaedics and Related Research, 445, 245-249.

[3] Lose, G. and Lindholm, P. (1986) Transient Painful Osteoporosis of the Hip in Pregnancy. International Journal of Gynecology \& Obstetrics, 24, 13-16. https://doi.org/10.1016/0020-7292(86)90017-2

[4] Cohen, L., Melamed, E., Lipkin, A., et al. (2007) Transient Osteoporosis of Pregnancy Complicated by a Pathologic Sub-Capital Hip Fracture. Journal of TraumaInjury Infection \& Critical Care, 62, 1281-1283. https://doi.org/10.1097/01.ta.0000198206.60286.2f

[5] Axt-Fliedner, R., Schneider, G., Seil, R., et al. (2001) Transient Bilateral Osteoporosis of the Hip in Pregnancy: A Case Report and Review of the Literature. Gynecologic and Obstetric Investigation, 51, 138-140. https://doi.org/10.1159/000052910

[6] Bermas, B.L. (2010) Musculoskeletal Changes and Pain during Pregnancy. UpToDate. Last updated on October 26.

http://www.uptodate.com/contents/musculoskeletal-changes-and-pain-during-preg nancy

[7] Curtiss, P.H. and Kincaid, W.E. (1959) Transitory Demineralization of the Hip in Pregnancy: A Report of Three Cases. The Journal of Bone \& Joint Surgery, 41, 1327-1333. https://doi.org/10.2106/00004623-195941070-00014

[8] Lequesne, M. (1968) Transient Osteoporosis of the Hip. Annals of the Rheumatic Diseases, 27, 463-471. https://doi.org/10.1136/ard.27.5.463

[9] Chowdhury, F.U., Robinson, P., Grainger, A.J., et al. (2006) Transient Regional Osteoporosis: A Rare Cause of Foot and Ankle Pain. Foot and Ankle Surgery, 12, 79-83. https://doi.org/10.1016/j.fas.2006.01.001

[10] Lloyd, J.M., Lewis, M. and Jones, A. (2006) Transient Osteoporosis of the Knee in Pregnancy: Case Report. Journal of Knee Surgery, 19, 121-123. https://doi.org/10.1055/s-0030-1248091

[11] Arayssi, T.K., Tawbi, H.A., Usta, I.M., et al. (2003) Calcitonin in the Treatment of Transient Osteoporosis of the Hip. Seminars in Arthritis \& Rheumatism, 32, 388-397. https://doi.org/10.1053/sarh.2002.50014

[12] La Montagna, G., Malesci, D., Tirri, R. and Valentini, G. (2005) Successful Neridronate Therapy in Transient Osteoporosis of the Hip. Clinical Rheumatology, 24, 67-69. https://doi.org/10.1007/s10067-004-0957-9 
[13] French, A.E., Kaplan, N., Lishner, M., et al. (2003) Taking Bisphosphonates during Pregnancy. Canadian Family Physician, 49, 1281-1282.

[14] Siminoski, K., Fitzgerald, A.A., Flesch, G., et al. (2000) Intravenous Pamidronate for Treatment of Reflex Sympathetic Dystrophy during Breast Feeding. Journal of Bone and Mineral Research, 15, 2052-2055. https://doi.org/10.1359/jbmr.2000.15.10.2052 